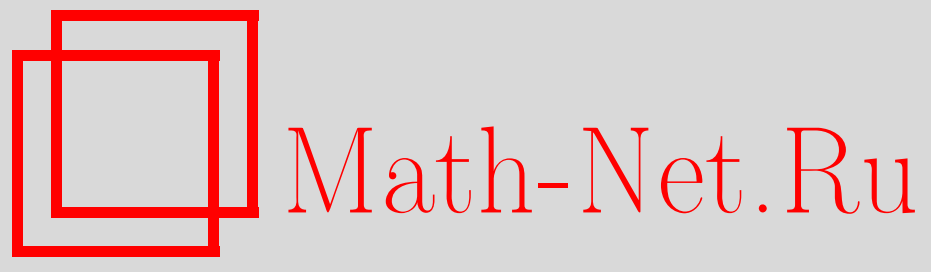

Е. М. Вечтомов, М. А. Лукин, Полукольца, являющиеся объединением кольца и полутела, УМН, 2008, том 63, выпуск 6, 159-160

DOI: https://doi.org/10.4213/rm9246

Использование Общероссийского математического портала Math-Net.Ru подразумевает, что вы прочитали и согласны с пользовательским соглашением http://www . mathnet.ru/rus/agreement

Параметры загрузки:

IP : 54.224 .135 .184

26 апреля 2023 г., 18:28:28

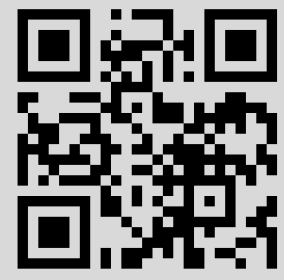




\section{Полукольца, являющиеся объединением кольца и полутела}

\section{Е. М. Вечтомов, М. А. Лукин}

1. Общие вопросы. Рассматриваются полукольца $S$ с единицей [1]. Если из делимого полукольца с квазитождеством $a+b=0 \Rightarrow a=0$ выбросить 0 , то получим алгебраическую структуру, называемую полутелом.

Обозначим через $r(S)$ множество всех элементов полукольца $S$, обладающих противоположным элементом, а через $U(S)$ - множество всех обратимых элементов из $S$. Очевидно, что $r(S)$ является кольцом относительно операций в $S$.

ОПРеДЕЛЕНИЕ 1 . Полукольцо $S$ назовем полукольцевым дизбюнктным объединением кольца $R$ и полутела $U$ и обозначим $R \dot{\cup} U$, если оно является объединением своих непересекающихся подмножеств $r(S)$ и $U(S)$, причем $r(S)$ есть кольцо $R, U(S)-$ полутело $U$.

При изучении указанных в заглавии полуколец можно ограничиться полукольцевыми дизъюнктными объединениями кольца и полутела, их изучение начато в [2].

Теорема 1. Кольцо $R$ входит в полукольцевое дизгюнктное объединение с некоторым полутелом тогда и только тогда, когда $R$ - радикальное по Джекобсону кольц,о, аддитивная группа которого является делимой абелевой группой без кручения.

Полутело $U$ называется зероидным, если существуют $a, b \in U$ такие, что $a+b=a$. Легко видеть, что незероидность полутела равносильна существованию на нем неединичной конгруэнции, фактор по которой есть сократимое полутело.

Теорема 2. Полутело $U$ входит в полуколъцевое дизгюнктное объединение с некоторым ненулевым кольцом тогда и только тогда, когда $U$ не является зероидным.

Теорема 3. Существуют неизоморфные полукольцевые дизбюнктные объединения кольца $R=\mu(0)$ и полутела $U={ }^{*}\left(\mathbb{R}^{+}\right) \times{ }^{*}\left(\mathbb{Q}^{+}\right)$, где $\mu(0)$ - монада нуля нестандартного расширения поля действительных чисел ${ }^{*} \mathbb{R}$.

Можно привести пример кольца и полутела, допускающих бесконечно много попарно неизоморфных полукольцевых дизъюнктных объединений.

Полутела, входящие в полукольцевое дизъюнктное объединение с данным кольцом, описаны в [2].

2. Строение колец, входящих в полукольцевое дизъюнктное объединение с данным полутелом. Фиксируем незероидное полутело $U$. Пусть для ненулевого кольца $R$ существует полукольцо $S=R \dot{\cup} U$. Рассмотрим условие $(*): 1+r \neq 1$ для любого ненулевого $r$ из $R$; оно равносильно импликации $1+r_{1}=1+r_{2} \Rightarrow r_{1}=r_{2}$ $\left(\forall r_{1}, r_{2} \in R\right)$.

Каждая конгруэнция $\rho$ на полутеле $U$ определяется любым своим классом, класс единицы конгруэнции $\rho$ называется ядром в $U$.

Ядро $K$ полутела $U$ назовем кольцевым, если: 1) $\forall u \in U \forall k \in K \exists t \in K \quad k u+1=$ $u+t$; 2) $\forall u \in U \forall k_{1}, k_{2} \in K\left(k_{1}+u=k_{2}+u \Rightarrow k_{1}=k_{2}\right)$.

Пусть $T(U)$ - кольцо разностей полутела $U$ и $h: U \rightarrow T(U)$ - естественный гомоморфизм, переводящий элемент $u \in U$ в класс $\overline{(u+1,1)}$.

Имеют место следующие утверждения.

УтвеРЖДЕниЕ 1. Если $K$ - кольцевое ядро полутела $U$, то гомоморфизм $h$ инбективен на $K$ и $h(K)=1+I$ для некоторого идеала $I$ кольца $T(U)$ такого, что существует $I \dot{\cup} U$ с условием (*). 
УтвеРЖДЕНИЕ 2. Колъцо $R$ входит в полукольцевое дизғюнктное объединение с условием $(*)$ с данным полутелом $U$ тогда и только тогда, когда $R$ изоморфно идеалу $1-h(K)$ кольца разностей $T(U)$ для некоторого кольцевого ядра $K$.

Доказательство. Пусть $S=R \cup \dot{U} U$ и в полукольце $S$ выполняется условие (*). Нетрудно видеть, что множество $1+R$ является классом единицы конгруэнции $\sigma$ на полутеле $U: u_{1} \sigma u_{2} \Leftrightarrow \exists r \in R u_{1}+r=u_{2}$. Докажем, что ядро $1+R$ - кольцевое. Возьмем $r, r_{1}, r_{2} \in R$ и $u, w \in U$. Поскольку $(1+r) u+1=u+(1+r u)$, то выполнено условие 1$)$ кольцевого ядра. Проверим 2). Пусть $\left(1+r_{1}\right) u+w=\left(1+r_{2}\right) u+w$, тогда $u+w+r_{1} u=u+w+r_{2} u$, или $1+r_{1} u(u+w)^{-1}=1+r_{2} u(u+w)^{-1}$, откуда в силу $(*)$ $r_{1} u(u+w)^{-1}=r_{2} u(u+w)^{-1}$, и $r_{1}=r_{2}$. Следовательно, $K=1+R$ - кольцевое ядро и $R \cong 1-h(K)$. Обратное утверждение следует из утверждения 1 .

УтвеРЖДЕнИЕ 3. Для полуколъцевого дизъюнктного объединения $S=R \dot{\cup} U$ множество $J(S)=\{r \in R: \exists u \in U \quad u+r=u\}$ является идеалом кольиа $R$, выдерживает умножение на элементы полутела $U$ и его аддитивная группа делима.

Tеорема 4. Пусть $S=R \cup \dot{U}$ для ненулевого кольца $R$ и полутела $U$. Тогда существует $(R / J(S)) \dot{\cup} U$ с условием $(*)$ и существует такое полукольцо $S_{1}=R \dot{\cup} U$, что $J\left(S_{1}\right)=\{r \in R: \forall u \in U \quad u+r=u\}$ и факторкольиа $R / J\left(S_{1}\right)$ и $R / J(S)$ изоморфны.

Доказательство. Рассмотрим $S=R \cup \dot{U}$. В силу утверждения $3 J(S)$ - идеал кольца $R$ и его аддитивная группа делима, поэтому она выделяется прямым слагаемым в группе $\langle R,+\rangle: J(S) \oplus P=R$. Очевидно, что $P$ выдерживает умножение на элементы полутела $U$. Аддитивная группа факторкольца $R / J(S)$ изоморфна $\langle P,+\rangle$, каждый элемент $R / J(S)$ имеет вид $[p]$ для единственного элемента $p \in P$. Положив $u \oplus[p]=[p] \oplus u=u+p, u \otimes[p]=[u \cdot p],[p] \otimes u=[p \cdot u]$ для элементов $u \in U, p \in P$, получим $(R / J) \cup \dot{\cup} U$. Поскольку $J(S) \cap P=\{0\}$, то это полукольцевое объединение удовлетворяет условию $(*)$.

Образуем новое полукольцевое дизъюнктное объединение $R \dot{\cup}_{1} U:(p+j) \oplus u=$ $u \oplus(p+j)=p+u,(p+j) \otimes u=p u+j u, u \otimes(p+j)=u p+u j$ при $p \in P, u \in U$, $j \in J(S)$. Операции заданы корректно, поскольку множества $P$ и $J(S)$ выдерживают умножение на элементы полутела $U$. В этом полукольцевом дизъюнктном объединении для каждого элемента $j \in J\left(S_{1}\right)$ и для каждого $u \in U$ выполняется равенство $j+u=u$. Теорема доказана.

3. Построение искомых колец $R$. В силу утверждений 1 и 2 строим все полукольца $S=T \cup \dot{U} U$ с условием $(*)$. Далее, опираясь на теорему 4 , берем всевозможные кольца $R$, для которых существует такой эпиморфизм $f: R \rightarrow T$ для одного из найденных колец $T$, что $R$ - унитарный полумодуль над $U$, Ker $f \subseteq \operatorname{Ann} R$ и $f(u r)=u f(r)$, $f(r u)=f(r) u$ для любых $u \in U$ и $r \in R$.

Если $R$ - кольцо с нулевым аннулятором и для данного полутела $U$ существует $S=R \cup \dot{\cup} U$, то $S$ удовлетворяет условию $(*)$. В силу утверждения 2 такие кольца $R$ имеют вид $1-K \subset T(U)$ по всем кольцевым ядрам $K$ в $U$.

\section{Список литературы}

[1] J. S. Golan, Semirings and their applications, Kluwer, Dordrecht, 1999. [2] М. А. Лукин, Чебышёвский сб., 6:4(16) (2005), 138-148.

\section{Е. М. Вечтомов (E. M. Vechtomov)}

Вятский государственный гуманитарный университет E-mail: vecht@mail.ru
Представлено А. В. Михалёвым Принято редколлегией 14.10 .2008

\section{М. А. Лукин (M. А. Lukin)}

Вятский государственный гуманитарный университет

E-mail: mathematic@vshu.kirov.ru 